\title{
Comparative Study of Polyamides from Bisacid A2*
}

\author{
Rudolph S. LenK, ${ }^{* *}$ John F. Fellers, and James L. White \\ Department of Chemical and Metallurgical Engineering, \\ The University of Tennessee, Knoxville, \\ Tennessee, 37916 U.S.A.
}

(Received April 6, 1976)

\begin{abstract}
Results of a study of the solution polycondensation of seven aromatic diamines with bisacid A2 are reported. The polymer of bisacid A2 with hexamethylene diamine is also synthesized. An evaluation of the polymers based upon wide angle X-ray scattering, infrared analysis, differential scanning calorimetry, thermogravimetric analysis, and mechanical properties has been carried out. Two of the polymers have been wet spun into fibers.

KEY WORDS Polycondensation / Fibers / Polyamide / Mechanical Properties / Bisacid A2 / X-Ray Diffraction / Aromatic Diamine / Calorimetry /
\end{abstract}

During the past decade, there has been considerable interest in the development of aromatic polyamides because of their high strength, modulus, and flame resistance..$^{1-3}$ This has led to various studies in our laboratories of the synthesis and formation of fibers from aromatic polyamides. $^{4,5}$ In 1971, Lenk reported the preparation ${ }^{6}$ of essentially pure bisacid A2:<smiles>CC(C)(c1ccc(OCC(=O)O)cc1)c1ccc(OCC(=O)O)cc1</smiles><smiles>Nc1ccc(N)cc1</smiles>

(I)<smiles>Cc1c(N)cccc1N</smiles>

(V)<smiles>Nc1cc(Cl)c(N)c(Cl)c1</smiles>

(II) and used it to synthesize various crosslinkable polyesters. ${ }^{7}$ Recently, the present authors ${ }^{8}$ have reported the synthesis of the product of bisacid A2 and $p$-phenylenediamine (which was called bisparamid A2) and discussed the properties of the resulting polymer and its fabrication into fibers. Ogata, Hosoda, and Suzuki ${ }^{9}$ have reported the synthesis of a polymer from bisacid A2 and hexamethylenediamine.

In this paper we report the preparation of aromatic polyamides based on bisacid $\mathrm{A} 2$ with several aromatic diamines and hexamethylene diamine. The diamines used were:<smiles>Nc1ccc(N)c([N+](=O)[O-])c1</smiles>

(III)<smiles>Nc1cccc(N)c1</smiles>

(IV)<smiles>Nc1ccc(Cl)c(N)c1</smiles>

(VII)

$$
\begin{gathered}
\mathrm{NH}_{2} \\
\vdots \\
\left(\mathrm{CH}_{2}\right)_{6} \\
\vdots \\
\mathrm{NH}_{2}
\end{gathered}
$$

(VIII)

\footnotetext{
* This is the second in a series of papers dealing with bisacid A2 polyamides and polyhydrazides.
}

** Permanent Address: Bank, London, England.
Polytechnic of the South 
R. S. Lenk, J. F. Fellers, and J. L. White

Table I. Polyamides synthesized from bisacid A2

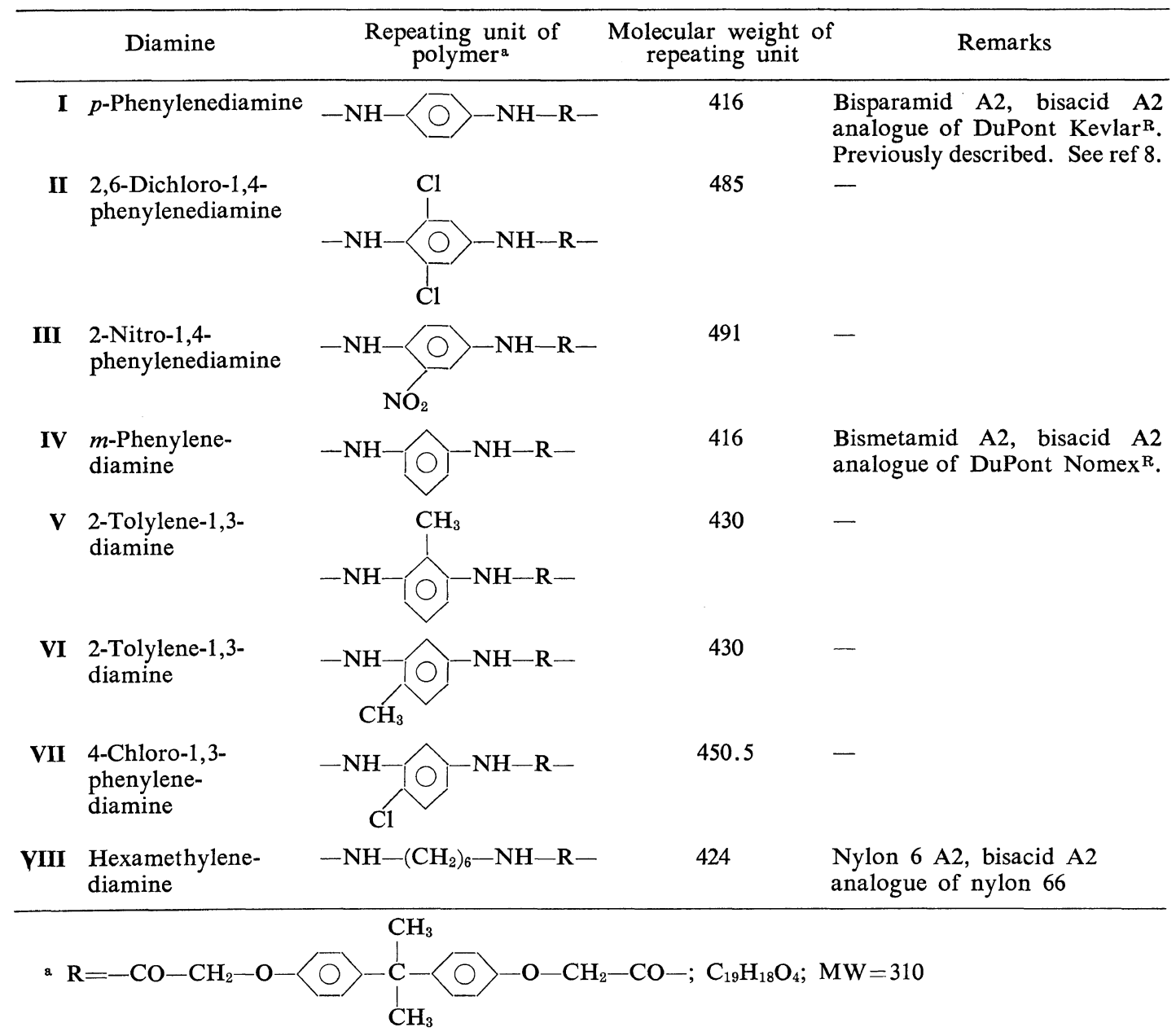

The polymers produced from bisacid A2 and these diamines are summarized in Table I. The synthesis involving monomers I-VII was carried out using low temperature solution polycondensation methods devised by Morgan and Kwolek. ${ }^{10-12}$ These methods have been previously applied in our laboratories for the preparation of aromatic polyamides. ${ }^{4,8}$ The polymer based on the aliphatic diamine VIII (hexamethylenediamine) was obtained by interfacial polymerization. ${ }^{11,13,14}$

\section{SYNTHESIS}

The methods of low temperature solution polycondensation $^{4,8-11}$ were applied to obtain the aromatic polyamides from diamines I-VII. Throughout this series, stoichiometric quantities of bischloride A2 and the appropriate diamine were reacted together in the manner described in our previous paper, ${ }^{8}$ which also contains an account of the detailed procedure for the synthesis and purification of bischloride A2. Stoichiometry was based on the effective molecular weight of bischloride A2, which was taken as 407.5. The solvent for the bischloride A2 was $\mathrm{CCl}_{4}$ and the diamine was dissolved in $\mathrm{N}$ methylpyrrolidone (NMP). Table II summarizes the polycondensation conditions, including the reactant concentration and the temperature at addition.

The inherent viscosities $\eta_{\mathrm{inh}}$ were measured 
Comparative Study of Polyamides from Bisacid A2

Table II. Bisacid A2-Aromatic diamine solution polycondensation conditions

\begin{tabular}{rcccc}
\hline $\begin{array}{c}\text { Diamine } \\
\text { type }\end{array}$ & $\begin{array}{c}\text { Batch } \\
\text { number }\end{array}$ & $\begin{array}{c}\text { Reaction } \\
\text { concentration, } \\
\text { wt } \%\end{array}$ & $\begin{array}{c}\text { Temperature } \\
\text { at addition, } \\
{ }^{\circ} \text { C }\end{array}$ & $\begin{array}{c}\text { Polymer } \\
\text { product, } \\
\eta_{\text {inh }}\end{array}$ \\
\hline I & $79,72^{\mathrm{a}}$ & $18.6,20.2$ & -30 & $1.50,2.03$ \\
II & 71 & 19.2 & -40 & 0.36 \\
III & 81 & 21 & -30 & 1.23 \\
IV & 80,73 & $18.2,21.4$ & -35 & $1.51,0.94$ \\
V & 69 & 18.2 & -25 & 0.56 \\
VI & 68 & 18.2 & -45 & 0.58 \\
VII & 70 & 18.5 & -30 & 1.27 \\
\hline
\end{tabular}

a Several conditions variously studied earlier ref 8 . The above is typical.

in $0.5-\%$ solution at $25^{\circ} \mathrm{C}$, the solvent being $5-\% \mathrm{LiCl}$ in dimethylacetamide (DMA). The results are contained in Table II. Generally, the conditions necessary for polymerization leading to high values for $\eta_{\mathrm{inh}}$ with regard to stoichiometry, concentration of reactants, and temperature at addition, as already established for diamine $\mathbf{I},{ }^{8}$ were also applied to the preparation of the bisacid $\mathrm{A} 2$ polyamides based upon the diamines II through VII.

Polymers based on diamine I (bisparamid A2) had $\eta_{\text {inh }}$ values of 1.5 to 2.0. As we have pointed out, ${ }^{8}$ this should be equivalent to a molecular weight of at least 13,000 to 18,000 . Diamine III led to polymers with $\eta_{\text {inh }}$ of 1.23 , which should have a slightly lower molecular weight. Polymers based on diamine IV (bismetamid A2) have an $\eta_{\text {inh }}$ of around 1.5 and diamine VI gave a polymer with $\eta_{\text {inh }}$ between 1.2 and 1.3. These should have molecular weights in the same range or even higher, because of the greater chain flexibility of the meta linkages. Polymers based on diamines II, $\mathbf{V}$, VI had $\eta_{\text {inh }}$ values ranging from 0.36 to 0.58 . These low values may have been due to impurities in the diamines.

The formation of an aliphatic polyamide from bisacid A2 and hexamethylene diamine by fusion of the previously prepared "nylon salt" has been accomplished by Ogata, et al., ${ }^{9}$ and Lenk ${ }^{15}$ earlier, but this only gave a polymer of $\eta_{\mathrm{inh}}=$ 0.38 (solvent: $m$-cresol). An attempt to obtain the same polyamide (nylon 6A2) by solution polymerization only gave $\eta_{\text {inh }}$ of 0.21 . Interfacial polymerization was then employed in an attempt to obtain a polymer with increased molecular weight.

The acid chloride may be dissolved in toluene or $\mathrm{CCl}_{4}$, depending on whether it is desired to have the organic phase above or below the interface; the diamine is dissolved in water containing sufficient $\mathrm{NaOH}$ to ensure neutralization of the $\mathrm{HCl}$ formed during the polycondensation. On bringing the solutions together at room temperature a pellicle of polymer quickly forms at the interface. In the case of nylon 6 A2 (the polyamide of bisacid A2 with the aliphatic hexamethylenediamine) this pellicle can be slowly but continuously pulled off, although it is weak and somewhat gelatinous. ${ }^{*}$

If the two phases are agitated together at high speed in a Waring Blender, an almost solid fibrous mass is obtained instantaneously; this is then filtered and washed with water. Filtration is slow because the fibrous mass holds both solvent and aqueous phase tenaciously and the two phases appear to obstruct the passage of each other through the filter paper. Addition of methanol has a drastic effect on aliphatic polyamides of bisacid A2; the fibrous mass collapses to a small volume and becomes swollen, though it does not actually dissolve. This makes filtration even more difficult. It was found best to filter as much as possible and then transfer the fibrous mass into boiling water. Kneading

* It is worth noting that aromatic diamines will also produce polyamides with bisacid A2 at the interface under analogous conditions, but the resulting pellicle is brittle and cannot be continuously pulled off; moreover, it has much lower $\eta_{\text {inh }}$ values (usually around 0.40) than those which can be attained by solution polymerization. 
in boiling water removed most of the occluded organic solvent as well as ionic impurities (principally $\mathrm{NaCl}$ ). The hot wet mass is rubbery elastic and reveals a fibrous structure when it is pulled.

\section{EVALUATION}

\section{Appearance}

The polymers as formed are an off-white to brown color. Films cast from various solvents are amber or brown in color. If films based on diamines I-VII are treated with formic acid, the films generally become white. This phenomenon has been discussed for polymers based on diamine I. $^{8}$ Films based on diamine VIII dissolve in formic acid.

Wide Angle X-Ray Studies (WAXS)

The films of the polymers based on the prime monomer types (I) and (IV) were studied using the methods of wide angle X-ray scattering. They were found to be amorphous. This has previously been discussed for the bisparamid $\mathrm{A} 2^{8}$ and is shown for bismetamid A2 (Figure 1). This is also the case with the formic acid treated films. An exception is found in the formic acid treated low molecular weight bisparamid A2 samples, which do crystallize. ${ }^{8}$

\section{Thermogravimetric Analysis (TGA)}

TGA studies were conducted on the polymers based on the prime monomer types (I) and (IV). These studies were conducted on the as-prepared polymer and also, the formic acid treated samples. The studies were carried out both in air and in a nitrogen atmosphere. Figure 2 depicts the results obtained. Basically, they show the wholly para-linked polymer to be slightly more stable than the one containing the meta diamine monomer unit. Also, as expected, all samples showed slightly greater stability in nitrogen as compared to air. The formic acid treated samples show a slight weight loss around $200^{\circ} \mathrm{C} \mathrm{(ca.} 1$ to $2 \%$ ) as compared to the untreated samples.

\section{Differential Scanning Calorimetry}

The various polymers synthesized from monomers I-VII were investigated using a PerkinElmer Differential Scanning Calorimeter. Polymers based on monomers I, III, IV, V, and

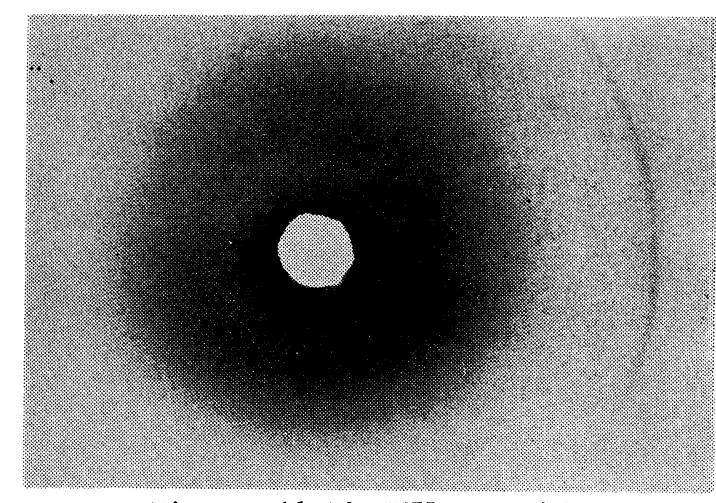

Bismetamid A2-73X, $\eta_{\text {inh }}=0.95$

Figure 1. WAXS pattern showing amorphous character of bismetamid A2 (after formic acid treatment). The WAXS pattern for bisparamid A2 is similar.
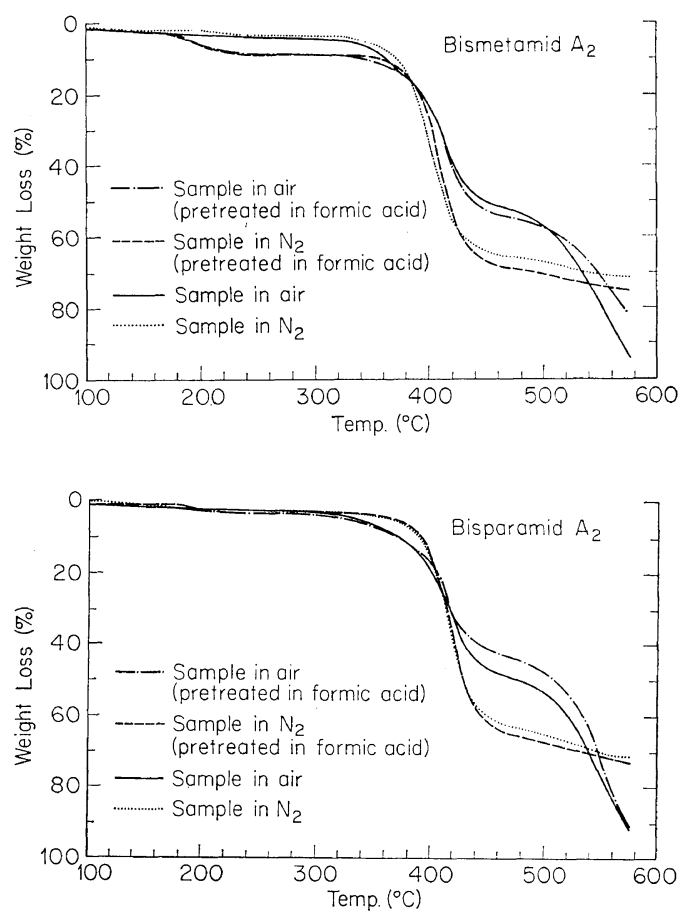

Figure 2. Thermogravimetric analysis study of bisparamid A2, showing weight loss as a function of temperature.

VI exhibited a small but consistent and characteristic small exothermic peak between $180^{\circ} \mathrm{C}$ and $190^{\circ} \mathrm{C}$. For polymers based on monomers II and VII, the peak was found at $210^{\circ} \mathrm{C}$ and 


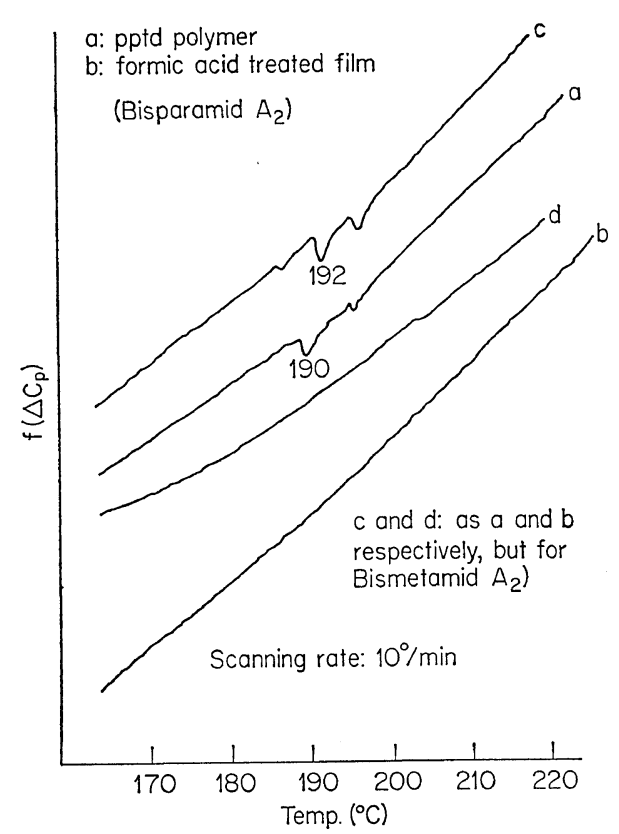

Figure 3. Differential scanning calorimeter 'scans' for bisparamid A2 and bismetamid A2, showing the influence of formic acid treatment. $208^{\circ} \mathrm{C}$ respectively. It should be noted that this feature of the DSC trace appears even in polymers of low $\eta_{\text {inh }}$ although it may occur some $8^{\circ} \mathrm{C}$ lower when $\eta_{\text {inh }}$ values of 0.5 are involved. Formic acid treated polymers do not exhibit this peak (see Figure 3). ${ }^{8}$

\section{Infrared (IR) Spectroscopy}

Since these polymers contain highly polar groups known to participate in hydrogen bonding, it was thought useful to examine the IR spectra of samples subjected to various conditioning procedures. Also, hydrogen bonding was thought to play an important role in the behavior of NOMEX $^{R}{ }^{16}$ Thus, films cast from dimethylacetamide solutions of the polymers made from III and VI were used for measuring IR spectra:

1. cast film and dried in vacuum oven for $2 \mathrm{hr}$ at $115^{\circ} \mathrm{C}$;

2. a section of film from 1 was washed in 90-\% formic acid and dried in a vacuum oven for $2 \mathrm{hr}$ at $115^{\circ} \mathrm{C}$;

3. a section of film from 2 was dried in a vacuum oven for 2 days at room temperature;

4. a section of film from 1 was dried in a vacuum oven for 2 days at $115^{\circ} \mathrm{C}$.

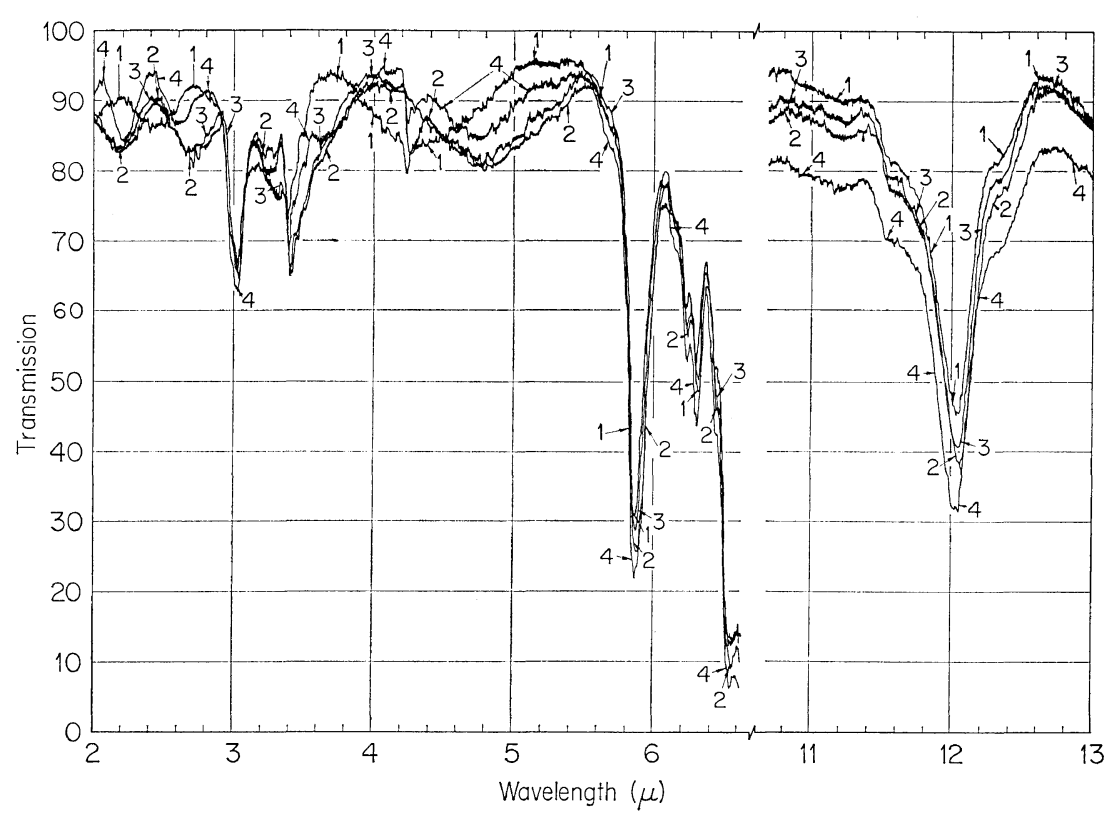

Figure 4. Comparative tracings of IR spectra of polymer from III subjected to treatment 1-4 described in the text. 
Table III. Mechanical properties of bisparamid A2, bismetamid A2, and nylon 6 A2 cast films

\begin{tabular}{|c|c|c|c|c|c|}
\hline & \multicolumn{2}{|c|}{ Bisparamid A2 $(\# 72)^{\mathrm{a}}$} & \multicolumn{2}{|c|}{ Bismetamid A2 $(\# 73)^{\mathrm{a}}$} & \multirow{2}{*}{$\frac{\text { Nylon } 6 \text { A2 }(\# 105)^{\mathrm{b}}}{\text { As-cast }}$} \\
\hline & As-cast & $\begin{array}{c}\text { Formic acid } \\
\text { treated }\end{array}$ & As-cast & $\begin{array}{l}\text { Formic acid } \\
\text { treated }\end{array}$ & \\
\hline $\begin{array}{l}\text { Failure stress, } \\
\text { dyn } \mathrm{cm}^{-2} \times 10^{-8}\end{array}$ & 5.66 & 3.45 & 5.80 & 4.77 & 3.32 \\
\hline Maximum strain, $\%$ & 3.0 & 9.5 & 2.6 & 2.7 & 1.93 \\
\hline $\begin{array}{l}\text { Initial modulus, } \\
\text { dyn } \mathrm{cm}^{-2} \times 10^{-10}\end{array}$ & 2.70 & 1.66 & 3.14 & 2.40 & 2.06 \\
\hline No. of test specimen & 7 & 8 & 8 & 8 & 8 \\
\hline
\end{tabular}

a Crosshead speed, $0.127 \mathrm{~cm} / \mathrm{min}$; gauge length, 6.0 to $7.0 ; 65-\%$ relative humidity.

b Crosshead speed, $0.508 \mathrm{~cm} / \mathrm{min}$; gauge length, $50 \mathrm{~cm} ; 65-\%$ relative humidity.

Polymer from III was subjected to steps 1 through 4 with IR spectra obtained for each step (see Figure 4). Polymer from VI was subjected to steps 1 and 2 with IR spectra then being obtained. A Beckman model IR IV was used.

The absorbances of the IR bands in the 2.8 to $3.3 \mu$ (amide hydrogen) region and the 5.8 to $6.0 \mu$ (carbonyl) region were followed using standard base line techniques to compare relative intensities. Several observations were made. First, the formic acid treatment produces changes in the amide hydrogen region from 2.8 to $3.3 \mu$ : specifically, compare the spectrum from procedure 1 to spectra from 2 and 3 shown in Figure 4. Next, it was noted that the change produced by formic acid is permanent, since the spectra from procedures 2 and 3 were equivalent. We consider that if the change were temporary and due to the presence of formic acid in the polymer, then continued storage under vacuum (procedure 3 ) would remove the formic acid and the spectrum would revert back to that observed in procedure 1. Such was not the case. Another observation is that extended thermal treatment as in procedure 4 produces some of the same changes as formic acid does, but the extent is not nearly as great.

\section{Mechanical Properties of Films}

Stress-strain tests were carried out on some of the films, notably bisparamid A2, bismetamid $\mathrm{A} 2$, and nylon $6 \mathrm{~A} 2$, using an Instron tensile testing machine. The engineering stress-strain curves are summarized in Figure 4 and in Table III. The bismetamid A2 and bisparamid A2

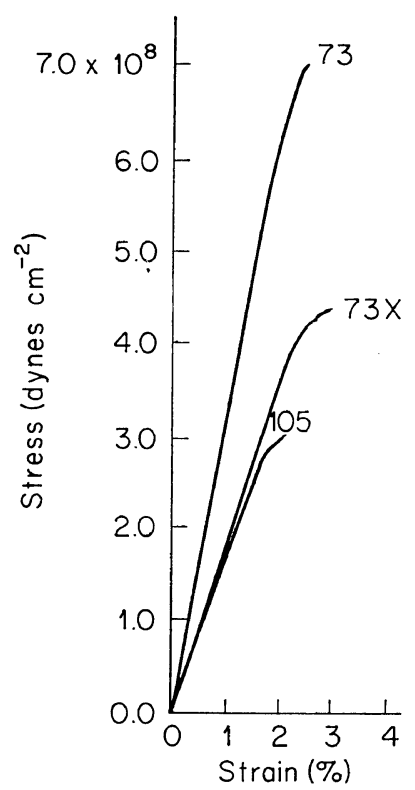

Figure 5. Stress-strain curves for bismetamid A2, bismetamid treated with formic acid, and nylon $6 \mathrm{~A} 2$.

have higher moduli and tensile strengths than the nylon $6 \mathrm{~A} 2$. The influence of formic acid treatment on the stress-strain curves of the bismetamid A2 is clearly discernible in Figure 5. It causes a lowering of modulus and tensile strength, but increases the elongation to break and tends to produce 'toughness.' Similar results were obtained for the bisparamid A2, as described earlier. ${ }^{8}$ In the case of nylon $6 \mathrm{~A} 2$, formic acid treatment is precluded owing to the solubility of the polymer in that medium, but 


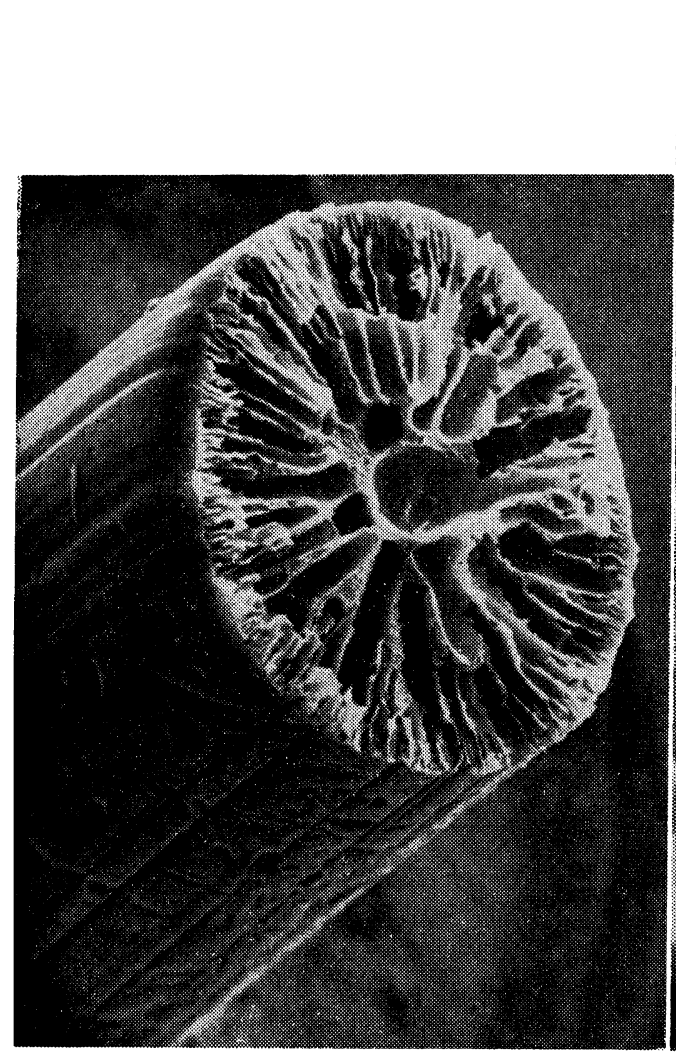

(a) $\times 200$

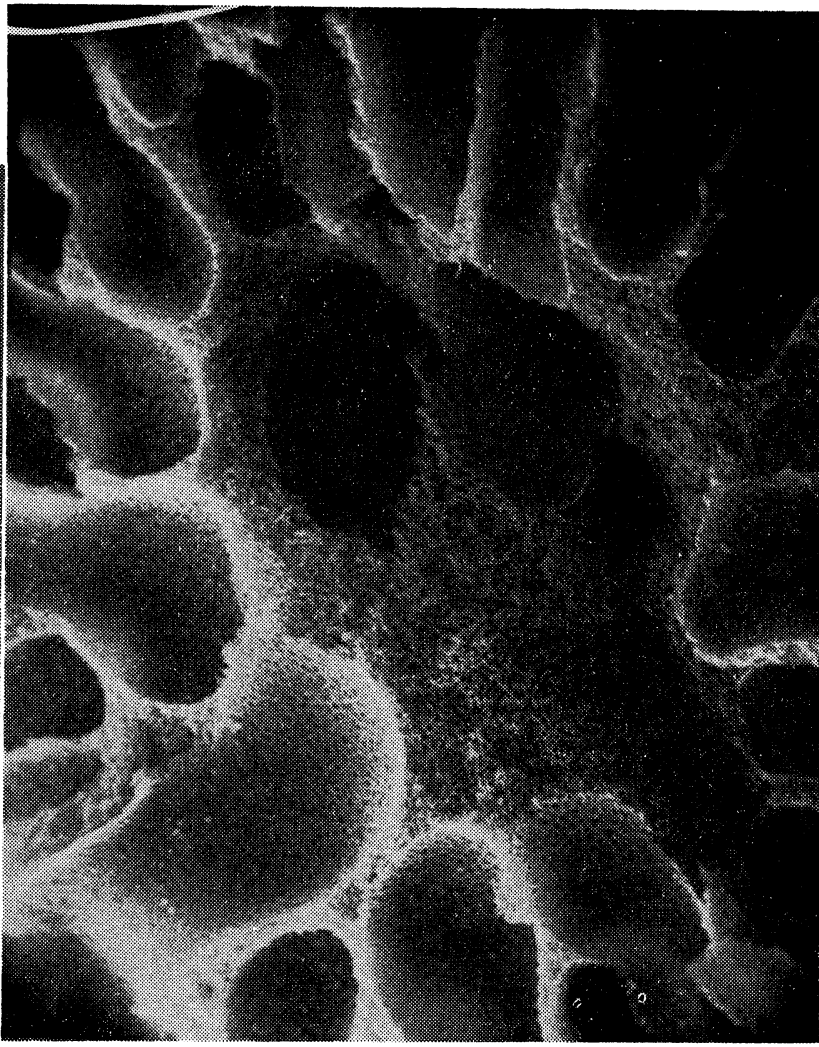

(b) $\times 500$

Figure 6. Scanning electron microscopy photomicrographs of wet spun bismetamid A2 fibers.

treatment with methanol seems to have a similar effect.

\section{Wet Spinning}

Fibers were wet spun from both bisparamid A2 and bismetamid A2. This was carried out using the apparatus and techniques of Hancock, Spruiell, and White. ${ }^{5}$ The studies for the bisparamid A2 have been discussed earlier. ${ }^{8}$ The bismetamid A2 was dissolved in DMA at a concentration of 10 to $12.5 \%$ and was spun through a water bath at room temperature. Scanning electron photomicrographs of the wet spun fiber are shown in Figure 6. Like the bisparamid A2 fibers, its cross-section shows a 'grapefruit' topography.

\section{DISCUSSION}

The work described in this paper has shown that the low-temperature solution polyconden- sation of bischloride A2 and p-phenylenediamine (diamine I) which affords bisparamid $\mathrm{A} 2^{8}$ is also applicable for making bismetamid A2, the $m$ phenylenediamine (diamine IV) analog of the first-mentioned polymer. It may also be employed for making polyamides from ring-substituted aromatic para and meta diamines, such as those exemplified by diamines II, III, V, VI, and VII.

Aliphatic nylons* can be obtained by fusion of the diamine A2 salt. This is the conventional method for making nylon 66 . It is also possible to obtain polymer by low temperature solution polymerization. However, neither of these methods has up to this point given polycondensates of high enough inherent viscosity for making fiber or even film. The best results for aliphatic polyamides of the nylon $n \mathrm{~A} 2$ type

* Nylons $n \mathrm{~A} 2$, where $n$ stands for the number of $\mathrm{C}$ atoms in the aliphatic diamine chain. 
are obtained by interfacial polymerization, though this method has many disadvantages and still does not afford polymer of very high $\eta_{\text {inh }}$. However, film forming polymer was obtained by interfacial polymerization, a method which, conversely, is not suitable for making bisparamids A2.

WAXS patterns show that the polymers from aromatic amines are typically amorphous, but that formic acid treatment may create conditions favorable to crystallization, provided the polymer is of low molecular weight.

Bisparamid A2 and bismetamid A2 may be cast into clear film, which turns white and opaque upon treatment with formic acid. Mechanical tests on the film show that as-cast films have tensile strengths of the order of $10^{8}$ dyn $\mathrm{cm}^{-2}$ at elongation rates of $0.127 \mathrm{~cm} \mathrm{~min}^{-1}$ $\left(23^{\circ} \mathrm{C}\right)$ and moduli of the order of $10^{10} \mathrm{dyn}^{-2}$. One notes that these values are higher than the nominal values obtained from aliphatic polyamides; however, they are also not nearly as high as those obtained for $p$-linked aromatic polyamides such as poly ( $p$-benzamide) or poly( $p$-phenylene terephthalamide). Black ${ }^{2}$ and Preston $^{3}$ have commented on the chemical structural requirements needed for ultra-high strength in wholly aromatic polyamides. Tensile tests on bismetamid A2 film gave similar results to those obtained on bisparamid A2 film in two major respects: (1) the magnitude of failure stress and initial modulus and (2) the effect of formic acid treatment in lowering both failure stress and modulus and tending to produce yield before break (tough failure). However, we did observe one major difference-formic acid treatment caused a large increase in the failure strain in bisparamid A2 film, but produced no significant change in the failure strain of bismetamid A2 film. We cannot at this stage attempt to explain this observation. The results are summarized in Table III. Typical stressstrain curves of bisparamid A2 film were reported earlier. ${ }^{8}$

Finally, we wish to draw attention to the exothermic peak in the DSC traces, which is characteristic for all bisaramids irrespective of the $\eta_{\mathrm{inh}}$ and which is found typically between 170 and $190^{\circ} \mathrm{C}$. This peak disappears upon treatments with formic acid. Sweeny ${ }^{16}$ has sug- gested that in the case of poly $(m$-phenylene isophthalamide) type polyamides a transformation may be brought about by heat or by treatment with suitable compounds such as formic acid or by high shear in solution and that the two types are intra- and intermolecularly hydrogen bond respectively. This hypothesis might also fit our case. It should be noted that the bisaramids, when freshly formed in solution, quickly become very viscous-indeed gelatinous and even rubbery, possibly under the influence of the $\mathrm{HCl}$ formed during the reaction. Dilution alone does not seem to break the gel, but neutralization or partial neutralization with $\mathrm{LiOH}$ does so.

Without regard to any specific hypothesis about inter-vs. intra-chain hydrogen bonding, our IR observations do bring some insight to the nature of the phenomenon. Conditioning treatments such as heating and formic acid washing do cause permanent changes in the hydrogen bonding of these polymers.

Acknowledgment. We would like to thank D. Coffin for his help in the IR measurements, T. Hancock for wet spinning of fibers, $Y$. Lee for wide angle X-ray scattering studies, and B. L. McGill for SEM photomicrograph investigations.

This research was supported in part by the National Science Foundation.

\section{REFERENCES}

1. W. B. Black and J. Preston in "Man-Made Fibers," Vol. 2, H. Mark, S. Atlas, and E. Cernia, Ed., John Wiley and Sons, Inc., New York, N.Y., 1968.

2. W. B. Black, Ed., "High Modulus Wholly Aromatic Fibers," J. Macromol. Sci., Chem., A7, 1-348 (1973); W. B. Black, MPT International Review of Science, Physical Science Series II, Macromolecular Chemistry Volume, C. E. H. Bawn, Ed., Butterworths, London, 1975 p 33-122.

3. J. Preston, Polym. Eng. Sci., 15, 199 (1975).

4. J. F. Fellers, R. Zdrahala, and E. Firer, "Block Copolymers of $\operatorname{Poly}(m$-phenylene isophthalamide) and Poly(ethylene oxide) or Poly(dimethyl siloxane): Synthesis and General Characteristics," University of Tennessee Polymer Science and Engineering Report No. 62, February 1976. 
5. T. Hancock, J. E. Spruiell, and J. L. White, "Wet Spinning of Aliphatic and Aromatic Polyamides," University of Tennessee Polymer Science and Engineering Report No. 54, January 1976.

6. R.S. Lenk, J. Appl. Polym. Sci., 15, 311, 1753 (1971).

7. R. S. Lenk, ibid., 15, 2211 (1971); 16, 129, 2921 (1972); 17, 821, 2245 (1973).

8. R. S. Lenk, J. L. White, and J. F. Fellers, "An Aromatic Polyamide from Bisacid A2 and $p$ Phenylene Diamine (Bisparamid A2): Synthesis Properties and Processing," University of Tennessee Polymer Science and Engineering Report No. 56, January 1976.
9. N. Ogata, Y. Hosoda, and G. Suzuki, Polym. J., 6, 412 (1974).

10. P. W. Morgan and S. L. Kwolek, J. Polym. Sci., 2, 181 (1964).

11. P. W. Morgan, "Condensation Polymers: By Interfacial and Solution Methods," Interscience Publishers Inc., New York, N. Y., 1965.

12. S. L. Kwolek, P. M. Morgan, and W. R. Sorenson, U. S. Patent 3063966 (1963).

13. E. L. Wittbecker and P. W. Morgan, J. Polym. Sci., 40, 289 (1959).

14. V. E. Shashoua and W. M. Eareckson, ibid., 20, 343 (1959).

15. R. S. Lenk, unpublished research (1974).

16. W. Sweeny, U. S. Patent 3287324 (1966). 